\title{
Gastrointestinal perforation during treatment with erlotinib plus bevacizumab in two patients with non-small cell lung cancer exhibiting epidermal growth factor receptor mutations: A case report
}

\author{
TEPPEI YAMAGUCHI ${ }^{1}$, YUSUKE GOTOH ${ }^{1}$, HIDEKAZU HATTORI ${ }^{2}$, \\ HIDETOSHI KATSUNO ${ }^{3}$ and KAZUYOSHI IMAIZUMI ${ }^{1}$
}

Departments of ${ }^{1}$ Respiratory Medicine, ${ }^{2}$ Radiology and ${ }^{3}$ Surgery, Fujita Health University, Toyoake, Aichi 470-1192, Japan

Received August 9, 2016; Accepted March 7, 2017

DOI: $10.3892 / \mathrm{ol} .2018 .8708$

\begin{abstract}
A previous randomized phase II study in patients with non-small cell lung cancer (NSCLC) identified that combination treatment with erlotinib plus bevacizumab prolonged progression-free survival compared with erlotinib alone. However, combination bevacizumab and erlotinib treatment generally increased the risk of severe adverse events, including hemorrhage, thrombosis, fistula formation and gastrointestinal perforation. The present report describes two patients with NSCLC harboring epidermal growth factor receptor (EGFR) mutations, who experienced gastrointestinal perforation associated with erlotinib plus bevacizumab combination therapy. The first patient, a 67-year-old male with stage IIIB lung adenocarcinoma harboring a L858R point mutation in EGFR exon 21, received concurrent chemoradiotherapy. However, seven months later, the patient experienced a relapse and was administered erlotinib plus bevacizumab treatment. A total of two months subsequent to commencing treatment, the patient developed a perforated duodenal ulcer. The second patient, a 66-year-old male with lung adenocarcinoma harboring a deletion in EGFR exon 19 and multiple pulmonary metastases, demonstrated a partial response to erlotinib plus bevacizumab treatment. A total of seven months subsequent to starting treatment, the patient experienced lower abdominal pain, and abdominal computed tomography confirmed a diagnosis of colocutaneous fistula complicating sigmoid diverticulitis. Following repair of the perforation, both patients were restarted on erlotinib treatment alone. Gastrointestinal perforation may
\end{abstract}

Correspondence to: Dr Teppei Yamaguchi, Department of Respiratory Medicine, Fujita Health University, 1-98 Dengakugakubo, Kutsukake-cho, Toyoake, Aichi 470-1192, Japan

E-mail: yteppei@fujita-hu.ac.jp

Key words: gastrointestinal perforation, bevacizumab, erlotinib, non-small cell lung cancer, epidermal growth factor receptor mutations be a potentially severe adverse event of erlotinib plus bevacizumab combination therapy, even in the absence of tumor metastasis in the abdomen.

\section{Introduction}

The development of molecular-targeted therapies has remarkably changed the management of patients with advanced non-small cell lung cancer (NSCLC) harboring specific genetic alterations, including epidermal growth factor receptor (EGFR) gene mutations and anaplastic lymphoma kinase rearrangements (1-7). Mutations in the EGFR gene are identified in 40-50\% of NSCLC in East Asian (8) and in $10-15 \%$ in Caucasian populations (9). Several phase III clinical trials have indicated that EGFR-tyrosine kinase inhibitors (TKIs) significantly improve median progression-free survival (PFS) compared with chemotherapy in patients with NSCLC harboring EGFR mutations (1-7). Tumors harboring these mutations, however, generally acquire resistance to EGFR-TKIs after a median time of 9-14 months, followed by disease progression. The ability of several combination regimens containing EGFR-TKIs to improve clinical outcomes in patients with NSCLC harboring EGFR mutations has therefore been tested (10-12).

Bevacizumab is a recombinant humanized monoclonal antibody that targets vascular endothelial growth factor, which has been demonstrated to inhibit the growth and maintenance of tumor blood vessels (13). The addition of bevacizumab to platinum doublet chemotherapy has been a standard of care for the first-line treatment of patients with advanced non-squamous NSCLC (14). The combination of EGFR-TKIs and bevacizumab is a promising treatment in patients with EGFR mutation-positive NSCLC (11,12). A multi-center randomized phase II trial reported that, compared with erlotinib alone, erlotinib plus bevacizumab combination therapy prolonged PFS in patients with NSCLC harboring EGFR mutations (12). Common side effects of bevacizumab include hypertension, proteinuria, thrombosis, hemorrhages and wound-healing complications (15). Gastrointestinal perforation is a relatively rare, but serious, adverse event associated with bevacizumab, which is reported in $0.3-3.2 \%$ 
of patients (15). To date, however, to the best of our knowledge this adverse event has not been reported during treatment of NSCLC patients with erlotinib plus bevacizumab. The present report describes the cases of two patients with advanced NSCLC, who experienced gastrointestinal perforations following erlotinib plus bevacizumab combination therapy.

\section{Case report}

Patient 1. A 67-year-old male observed to have an enlarged right hilar lymph node during a medical check-up was admitted to Fujita Health University Hospital (Toyoake, Aichi, Japan) in July 2014. The patient underwent a lymph node biopsy and was diagnosed with lung adenocarcinoma. Computed tomography (CT) revealed a primary tumor in the right lower lobe, which was diagnosed as stage IIIB (cT1aN3M0) according to the 7 th edition TNM classification (16). A total of $80 \mathrm{mg} / \mathrm{m}^{2}$ cisplatin (Nichi-Iko Pharmaceutical Co., Ltd., Toyama, Japan) on day 1, plus $20 \mathrm{mg} / \mathrm{m}^{2}$ vinorelbine (Kyowa Hakko Kirin Co., Ltd., Tokyo, Japan) on day 1 and 8 of each 4 week cycle concurrent with radiotherapy (60 Gy in 30 fractions) were immediately administered, with a CT scan of the chest indicating a partial response (PR) following two cycles of combination chemotherapy. However, seven months later the disease relapsed in the lymph nodes of the patient's right neck. Positron emission tomography revealed fluorodeoxyglucose uptake only in the right lower lobe and neck lymph nodes (Fig. 1). Mutation analysis of the tumor biopsy revealed a L858R point mutation in exon 21 of EGFR, and the patient was administered treatment with $150 \mathrm{mg} /$ day erlotinib, plus $15 \mathrm{mg} / \mathrm{kg}$ bevacizumab (both Chugai Pharmaceutical Co. Ltd., Tokyo, Japan) once every three weeks, starting on day 1 . A Grade 1 skin rash (Common Terminology Criteria for Adverse Events Version 4.0; CTCAE v4.0) (17) was the only side effect during the first two treatment cycles.

A total of two months later, the patient was admitted to Fujita Health University Hospital due to acute upper abdominal pain. Physical examination revealed generalized abdominal guarding and rebound tenderness. Abdominal CT showed spots of free intraperitoneal air below the ventral abdominal wall (Fig. 2). These clinical findings suggested a gastrointestinal perforation. Explorative laparoscopy revealed a perforated duodenal ulcer, and laparoscopic repair was performed. The patient resumed erlotinib treatment alone, without bevacizumab, three weeks following emergency surgery. The treatment was continued until the diagnosis of disease progression, and progressive disease in neck lymph node metastasis was confirmed at nine months.

The patient gave informed consent for the publication of their data in the present report.

Patient 2. A 66-year-old male was referred to Fujita Health University Hospital with increasing exertional dyspnea in March 2015. A chest CT scan revealed a mass in the left lower lobe of the lung, with multiple lung metastases, carcinomatous lymphangiosis and malignant pleural effusion. The patient was diagnosed with lung adenocarcinoma (cT4N3M1a, the 7th edition TNM classification; Fig. 3) (16). Sequencing analysis of the patient's pleural effusion revealed a deletion in EGFR exon 19 (E746-A750). The patient's previous medical history included paroxysmal atrial fibrillation, a risk factor for thromboembolism, and an inguinal hernia repair three years earlier. The patient's pleural effusion was uncontrollable following pleural drainage and pleurodesis. Following an explanation of the risk and benefits of combination bevacizumab treatment, the patient provided written informed consent for treatment with erlotinib (150 mg/day) plus bevacizumab $(15 \mathrm{mg} / \mathrm{kg}$ every 3 weeks). Following the commencement of combination treatment, the patient demonstrated marked improvement in multiple pulmonary carcinomatous lymphangiosis and exertional dyspnea.

Following five months of treatment, the patient experienced a grade 3 skin rash (CTCAE v4.0) (17), and the erlotinib dose was reduced to $100 \mathrm{mg} /$ day. A total of seven months following initiation of combination therapy, the patient experienced persistent lower abdominal pain for several days and was admitted to the Fujita Health University Hospital. Abdominal CT scanning confirmed the diagnosis of colon perforation and colocutaneous fistula complicating sigmoid diverticulitis (Fig. 4). The patient underwent surgical drainage and ileostomy, and was re-started on erlotinib treatment alone, without bevacizumab, six weeks following the surgery. The treatment was continued for an additional 18 weeks, and disease progression was observed in April 2016.

The patient gave informed consent for the publication of their data in the present report.

\section{Discussion}

To the best of our knowledge, these are the first reports of gastrointestinal perforation associated with erlotinib plus bevacizumab therapy in patients with NSCLC. The previous randomized phase II JO25567 trial demonstrated that treatment with erlotinib plus bevacizumab increased median PFS by more than 6 months, compared with erlotinib alone, in patients with activating EGFR mutation-positive NSCLC (12). However, the addition of bevacizumab to erlotinib may increase the numbers of potentially life-threatening adverse events, including bleeding, arterial and venous thromboembolic events, wound-healing complications, and gastrointestinal perforations (15). A meta-analysis of randomized controlled trials that included bevacizumab revealed that bevacizumab was associated with an increased risk of gastrointestinal perforation (18). The mechanism by which bevacizumab induces bowel perforation has not been determined, but may be associated with delayed wound healing (19).

In the present report, Patient 1 experienced a sudden onset of duodenal perforation without use of aspirin or other non-steroidal anti-inflammatory drugs. Had the patient not been exposed to bevacizumab, the duodenal ulcer may not have perforated. The patient was not taking any proton pump inhibitor (PPI) or $\mathrm{H}_{2}$ antagonists. Although these agents increase the $\mathrm{pH}$ of the upper gastrointestinal tract and heal ulcers, they reduce the solubility of erlotinib, thereby reducing its bioavailability $(20,21)$. Thus, acid-reducing agents are generally not administered to patients taking EGFR-TKIs.

The acidic beverage Coca-Cola (CC; The Coca-Cola Company, Atlanta, GA, USA) was recently demonstrated to improve the bioavailability of erlotinib in patients treated with a PPI (22). When erlotinib is taken with CC instead 


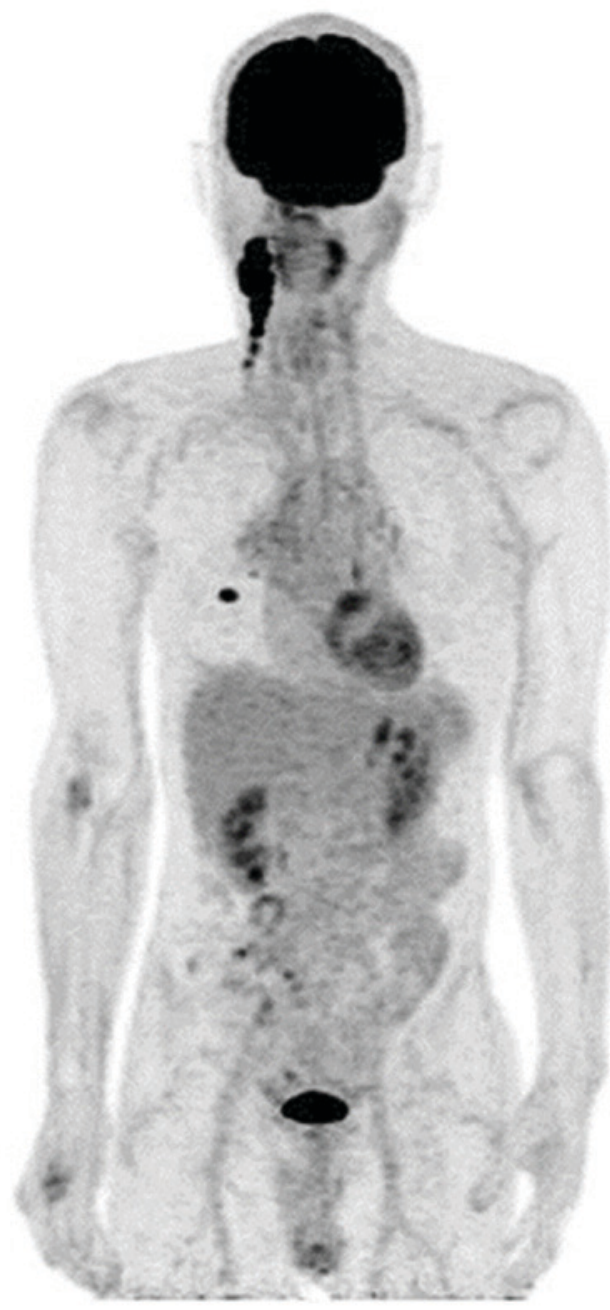

Figure 1. Whole-body maximum intensity projection of positron emission tomography data in Patient 1 , revealing a primary right lower lobe lung nodule and right side cervical lymph node metastases.

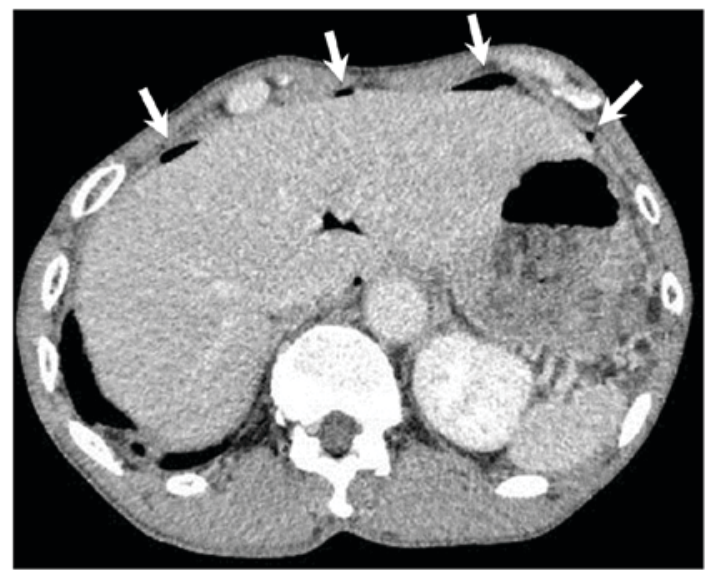

Figure 2. Computed tomography scan in Patient 1 revealing free air (white arrows) in the anterior peritoneal space due to perforation of a duodenal ulcer.

of water, the stomach is temporarily acidified, thereby increasing the absorption rate of erlotinib in patients taking esomeprazole. Taking erlotinib with CC instead of water is therefore recommended for patients being treated with erlotinib and a PPI (22).

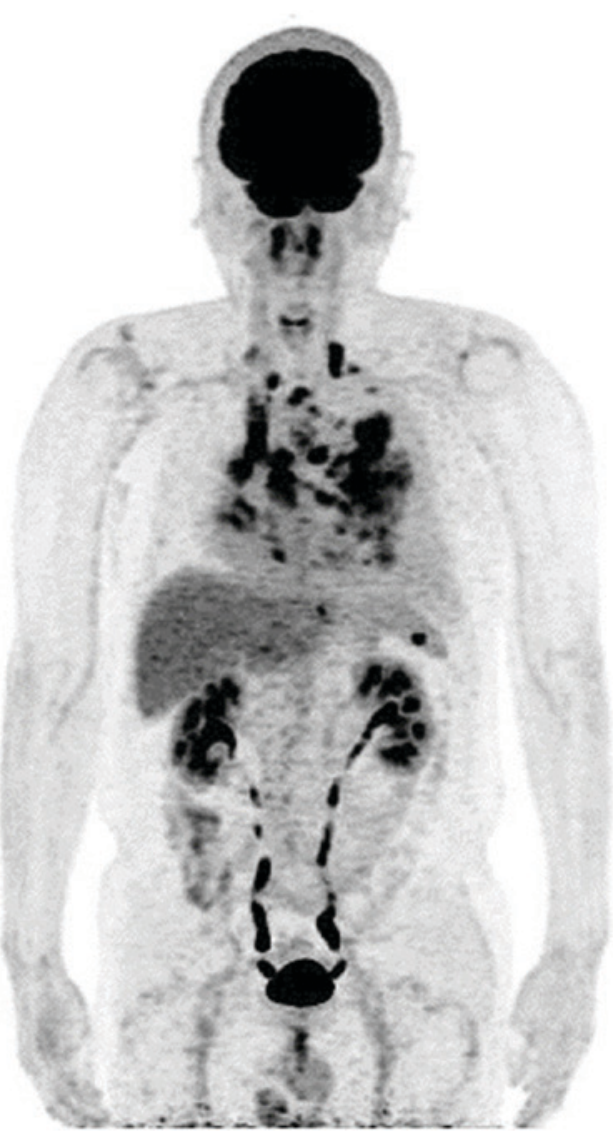

Figure 3. Whole-body maximum intensity projection of positron emission tomography data in Patient 2, revealing widespread pulmonary nodules and mediastinal lymph node metastases.

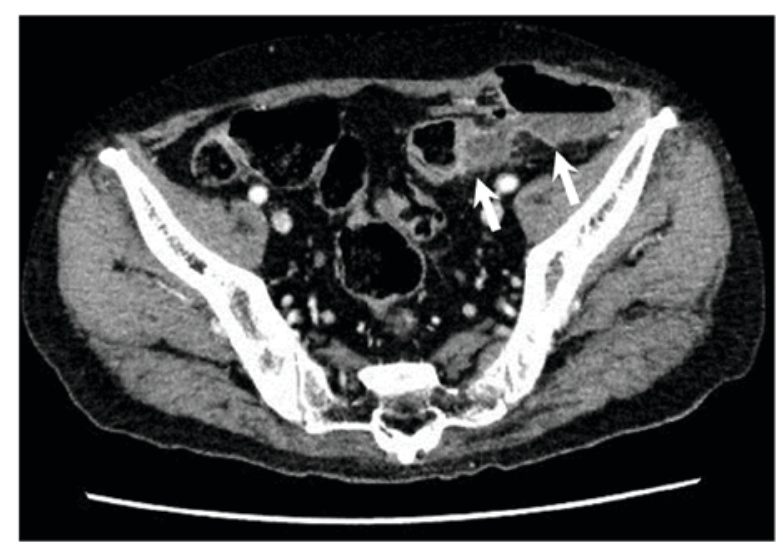

Figure 4. Computed tomography scan of Patient 2 revealing a colocutaneous fistula, a communication between diverticulitis of the sigmoid colon and subcutaneous tissue in the left iliac region (white arrows).

Treatment with bevacizumab may lead to necrosis of bowel tumors, which may result in bowel perforation (19). Colon perforation and colocutaneous fistula in Patient 2 were complications of sigmoid diverticulitis. As this patient underwent hernia repair 3 years previously, there may have been some weakness in the sigmoid colon near the suture site. However, the patient did not exhibit subjective symptoms prior to commencing erlotinib plus bevacizumab therapy, suggesting that this treatment had a significant effect on colon perforation. 
The incidence of gastrointestinal perforation has been reported to vary by tumor type, being increased in patients with colorectal carcinoma and renal cell cancer (18). These findings suggest that tumor location in the abdominal cavity may be a risk factor for gastrointestinal perforation. Bevacizumab-induced necrosis of these tumors may lead to gastrointestinal perforation. However, there was no evidence that intra-abdominal tumor in either of the present patients triggered perforation.

Bevacizumab is frequently combined with cytotoxic regimens, including taxanes, which may be associated with bowel inflammation and necrosis (23). Inflammation may be induced by tumor-promoting agents, mechanical stimulation or chemotherapy. Delayed wound healing associated with bevacizumab may lead to the perforation of bowels with acute inflammation (19). However, EGFR-TKIs have been associated with diarrhea. A phase II study of erlotinib plus bevacizumab in 13 patients with recurrent ovarian, primary peritoneal and fallopian tube cancer reported that two of these patients experienced gastrointestinal perforation (24). These findings suggested that erlotinib-induced diarrhea may increase the risk of bowel perforation.

In conclusion, gastrointestinal perforation is a potentially fatal adverse event associated with bevacizumab combination therapy, and may occur even in the absence of tumor spread in the abdomen. Although gastrointestinal perforation is difficult to predict, careful patient monitoring may prevent a fatal outcome.

\section{Acknowledgements}

Not applicable.

\section{Funding}

No funding was received.

\section{Availability of data and materials}

All data generated or analyzed during this study are included in this published article.

\section{Authors' contributions}

TY designed the study and wrote the initial draft of the manuscript with support from YG, HK and KI. HH contributed to analysis computed tomography and assisted in the preparation of the manuscript. All authors approved the final version of the manuscript and agreed to be accountable for all aspects of the work in ensuring that questions associated with the accuracy or integrity of any part of the study are appropriately investigated and resolved.

\section{Ethics approval and consent to participate}

Not applicable.

\section{Consent for publication}

The patient provided written informed consent for publication of this case report, and the privacy policy was fully explained.

\section{Competing interests}

The authors declared that they have no competing interests.

\section{References}

1. Mok TS, Wu YL, Thongprasert S, Yang CH, Chu DT, Saijo N, Sunpaweravong P, Han B, Margono B, Ichinose Y, et al: Gefitinib or carboplatin-paclitaxel in pulmonary adenocarcinoma. N Engl J Med 361: 947-957, 2009.

2. Mitsudomi T, Morita S, Yatabe Y, Negoro S, Okamoto I, Tsurutani J, Seto T, Satouchi M, Tada H, Hirashima T, et al: Gefitinib versus cisplatin plus docetaxel in patients with non-small-cell lung cancer harbouring mutations of the epidermal growth factor receptor (WJTOG3405): An open label, randomised phase 3 trial. Lancet Oncol 11: 121-128, 2010.

3. Maemondo M, Inoue A, Kobayashi K, Sugawara S, Oizumi S, Isobe H, Gemma A, Harada M, Yoshizawa H, Kinoshita I, et al: Gefitinib or chemotherapy for non-small-cell lung cancer with mutated EGFR. N Engl J Med 362: 2380-2388, 2010.

4. Zhou C, Wu YL, Chen G, Feng J, Liu XQ, Wang C, Zhang S, Wang J, Zhou S, Ren S, et al: Erlotinib versus chemotherapy as first-line treatment for patients with advanced EGFR mutation-positive non-small-cell lung cancer (OPTIMAL, CTONG-0802): A multicentre, open-label, randomised, phase 3 study. Lancet Oncol 12: 735-742, 2011.

5. Rosell R, Carcereny E, Gervais R, Vergnenegre A, Massuti B, Felip E,Palmero R, Garcia-Gomez R, Pallares C, Sanchez JM, et al: Erlotinib versus standard chemotherapy as first-line treatment for european patients with advanced EGFR mutations-positive non-small cell lung cancer (EURTAC) a multicenter, open-label, randomized phase 3 trial. Lancet Oncol 13: 239-246, 2012.

6. Sequist LV, Yang JC, Yamamoto N, O'Byrne K, Hirsh V, Mok T, Geater SL, Orlov S, Tsai CM, Boyer M, et al: Phase III study of afatinib or cisplatin plus pemetrexed in patients with metastatic lung adenocarcinoma with EGFR mutations. J Clin Oncol 31: 3327-3334, 2013

7. Wu YL, Zhou C, Hu CP, Feng J, Lu S, Huang Y, Li W, Hou M, Shi JH, Lee KY, et al: Afatinib versus cisplatin plus gemcitabine for first-line treatment of Asian patients with advanced non-small-cell lung cancer harbouring EGFR mutations (LUX-Lung 6): An open-label, randomised phase 3 trial. Lancet Oncol 15: 213-222, 2014.

8. Shi Y, Au JS, Thongprasert S, Srinivasan S, Tsai CM, Khoa MT, Heeroma K, Itoh Y, Cornelio G and Yang PC: A prospective, molecular epidemiology study of EGFR mutations in Asian patients with advanced non-small-cell lung cancer of adenocarcinoma histology (PIONEER). J Thorac Oncol 9: 154-162, 2014.

9. Rosell R, Moran T, Queralt C, Porta R, Cardenal F, Camps C, Majem M, Lopez-Vivanco G, Isla D, Provencio M, et al: Screening for epidermal growth factor receptor mutations in lung cancer. $\mathrm{N}$ Engl J Med 361: 958-967, 2009.

10. Sugawara S, Oizumi S, Minato K, Harada T, Inoue A, Fujita Y, Maemondo M, Yoshizawa H, Ito K, Gemma A, et al: Randomized phase II study of concurrent versus sequential alternating gefitinib and chemotherapy in previously untreated non-small cell lung cancer with sensitive EGFR mutations: NEJ005/TCOG0902. Ann Oncol 26: 888-894, 2015.

11. Ichihara E, Hotta K, Nogami N, Kuyama S, Kishino D, Fujii M, Kozuki T, Tabata M, Harada D, Chikamori K, et al: Phase II trial of gefitinib in combination with bevacizumab as first-line therapy for advanced non-small cell lung cancer with activating EGFR gene mutations: The okayama lung cancer study group trial 1001. J Thorac Oncol 10: 486-491, 2015.

12. Seto T, Kato T, Nishio M, Goto K, Atagi S, Hosomi $\mathrm{Y}$, Yamamoto N, Hida T, Maemondo M, Nakagawa K, et al: Erlotinib alone or with bevacizumab as first-line therapy in patients with advanced non-squamous non-small-cell lung cancer harbouring EGFR mutations (JO25567): An open-label, randomised, multicentre, phase 2 study. Lancet Oncol 15: 1236-1244, 2014.

13. Wedam SB, Low JA, Yang SX, Chow CK, Choyke P, Danforth D, Hewitt SM, Berman A, Steinberg SM, Liewehr DJ, et al: Antiangiogenic and antitumor effects of bevacizumab in patients with inflammatory and locally advanced breast cancer. J Clin Oncol 24: 769-777, 2006.

14. Sandler A, Gray R, Perry MC, Brahmer J, Schiller JH, Dowlati A, Lilenbaum R and Johnson DH: Paclitaxel-carboplatin alone or with bevacizumab for non-small-cell lung cancer. N Engl J Med 355: 2542-2550, 2006. 
15. US Food and Drug Administration, Center for Drug Evaluation and Research: AVASTIN (bevacizumab) solution for intravenous infusion [product label]. https://www.accessdata.fda.gov/drugsatfda_docs/label/2017/761028s000lbl.pdf. Accessed April 22, 2018.

16. Vallières E, Shepherd FA, Crowley J, Van Houtte P, Postmus PE, Carney D, Chansky K, Shaikh Z and Goldstraw P; International Association for the Study of Lung Cancer International Staging Committee and Participating Institutions: The IASLC lung cancer staging project: Proposals regarding the relevance of TNM in the pathologic staging of small cell lung cancer in the forthcoming (seventh) edition of the TNM classification for lung cancer. J Thorac Oncol 4: 1049-1059, 2009.

17. Common Terminology Criteria for Adverse Events (CTCAE), Version 4.0 Published: May28, 2009 (v4.03: June 14, 2010). U.S. Department of Health and Human Services, National Institute of Health National Cancer Institute. Available at https:// www.eortc.be/services/doc/ctc/CTCAE_4.03_2010-06-14 QuickReference_5x7.pdf. Accessed April 22,2018.

18. Hapani S, Chu D and Wu S: Risk of gastrointestinal perforation in patients with cancer treated with bevacizumab: A meta-analysis. Lancet Oncol 10: 559-568, 2009.

19. Gordon CR, Rojavin Y, Patel M, Zins JE, Grana G, Kann B, Simons R and Atabek U: A review on bevacizumab and surgical wound healing: An important warning to all surgeons. Ann Plast Surg 62: 707-709, 2009.
20. US Food and Drug Administration, Center for Drug Evaluation and Research: Tarceva (erlotinib) tablets for oral use [product label]. https://www.accessdata.fda.gov/drugsatfda_docs/ label/2014/021743s019lbl.pdf. Accessed April 22, 2018.

21. Chu MP, Ghosh S, Chambers CR, Basappa N, Butts CA, Chu Q, Fenton D, Joy AA, Sangha R, Smylie M and Sawyer MB: Gastric acid suppression is associated with decreased erlotinib efficacy in non-small-cell lung cancer. Clin Lung Cancer 16: 33-39, 2015

22. van Leeuwen RW, Peric R, Hussaarts KG, Kienhuis E, IJzerman NS, de Bruijn $\mathrm{P}$, van der Leest $\mathrm{C}$, Codrington $\mathrm{H}$, Kloover JS, van der Holt B, et al: Influence of the acidic beverage cola on the absorption of erlotinib in patients with non-small-cell lung cancer. J Clin Oncol 34: 1309-1314, 2016.

23. Seewaldt VL, Cain JM, Goff BA, Tamimi H, Greer B and Figge D: A retrospective review of paclitaxel-associated gastrointestinal necrosis in patients with epithelial ovarian cancer. Gynecol Oncol 67: 137-140, 1997.

24. Nimeiri HS, Oza AM, Morgan RJ, Friberg G, Kasza K, Faoro L, Salgia R, Stadler WM, Vokes EE, Fleming GF, et al: Efficacy and safety of bevacizumab plus erlotinib for patients with recurrent ovarian, primary peritoneal, and fallopian tube cancer: A trial of the Chicago, PMH, and California Phase II Consortia. Gynecol Oncol 110: 49-55, 2008. 